\title{
Randomly Branched Polymers. I. Hydrodynamic Properties*
}

\author{
Michio Kurata, ${ }^{* *}$ Mitsuo Abe, ${ }^{* * *}$ Masamichi Iwama, ${ }^{* * *}$ \\ and Masahiro Matsushima**** \\ Institute for Chemical Research, Kyoto University, \\ Uji, Kyoto 511, Japan.
}

(Received February 25, 1972)

\begin{abstract}
A sample of copolymer of styrene and 1,4-diviny1-2,3,5,6-tetrachlorobenzene was prepared and fractionated. Using the fractionated copolymers, effects of chain branching on the intrinsic viscosity and sedimentation coefficient were studied experimentally. It was found that in both theta and good solvents, the intrinsic viscosity of the branched polymers obeyed a new semiempirical relationship, $[\eta]_{b}=g^{0.6}[\eta]_{1}$. Here $[\eta]_{1}$ is the intrinsic viscosity of a linear polymer having the same molecular weight as the branched one and $g$ is the contraction factor which is defined as the ratio of the mean-square radii of gyration between the branched and linear polymers, i.e., $g=\left\langle S^{2}\right\rangle_{\mathrm{b}} /\left\langle S^{2}\right\rangle_{1}$. It was also found that the sedimentation coefficient in theta solvent agreed with the theoretical value obtained by Kurata and Fukatsu. The degree of branching estimated from the intrinsic viscosity or sedimentation coefficient was in close agreement with the value estimated from consideration of the copolymerization kinetics.
\end{abstract}

KEY WORDS Branched Polymer / Polystyrene / Degree of Chain Branching / Intrinsic Viscosity / Sedimentation Coefficient / MeanSquare Radius of Gyration / Theta Solvent / Zimm-Stockmayer Theory / Kurata-Fukatsu Theory /

In this series of papers, we report the results of a systematic study which has been performed to develop a quick and simple method of estimating the degree of long-chain branching in a polymer molecule.

According to Zimm and Stockmayer, ${ }^{1}$ the effect of chain branching on the molecular dimension in solution is expressed as

$$
\begin{gathered}
\left\langle S^{2}\right\rangle_{\mathrm{b}}=\left\langle S^{2}\right\rangle_{1} g \\
g=\left[(1+\lambda M / 6)^{1 / 2}+(4 / 3 \pi) \lambda M\right]^{-1 / 2}
\end{gathered}
$$

where $\left\langle S^{2}\right\rangle_{\mathrm{b}}$ and $\left\langle S^{2}\right\rangle_{l}$ are the mean-square radii

* Part of the doctoral thesis of M. Abe presented to Kyoto University in 1968 . The main part of this work was reported at the SPSJ 16th Symposium on Macromolecules, Fukuoka, October, 1967.

** To whom correspondence should be addressed.

*** Present address: Research Laboratory, Japan Synthetic Rubber Co., Ltd., Mochiizaka, Ikuta, Kawasaki, Japan.

**** Present address: Mitsubishi Rayon Co., Ltd., Ohtake, Hiroshima, Japan. of gyration for a branched molecule and a linear one, respectively, both of molecular weight $M$, and $\lambda$ is the average density of branch units which are assumed, in eq 2 , to be tetra-functional. Accordingly, $\lambda M$ represents the average number of branch units involved in a molecule. This eq 1 offers the possibility of estimating the degree of branching $\lambda$ from light-scattering measurements. The method is straightforward in principle, but its application is seriously limited by the difficulty in preparing a branched polymer sample with a narrow distribution of molecular weights. For ordinary samples of branched polymers, the branching effect on $\left\langle S^{2}\right\rangle$ is often screened with the effect of molecular weight distribution, ${ }^{2}$ and it becomes rather difficult to estimate these effects separately from each other. ${ }^{3}$

The hydrodynamic properties of polymer solutions are less sensitive to the molecular weight distribution than is the radius of gyration, still they are appreciably dependent on the shape of molecule. Thus, a more practical method for 
estimating the branching degree than the above could be developed in relation to the hydrodynamic properties, especially with respect to the intrinsic viscosity. A theory for the intrinsic viscosity of a branched polymer has been developed by $\mathrm{Zimm}$ and $\mathrm{Kilb}^{4}{ }^{4}$ but the type of branching treated has been limited to the socalled star type. A theory of the sedimentation coefficient has been also presented by Kurata and Fukatsu, which is applicable to various types of branching including the randam type. ${ }^{5}$ However, no experimental test has been reported yet. Thus, we found it worthwhile to study experimentally the effect of chain branching on the hydrodynamic properties of solutions and to establish a semiempirical equation of intrinsic viscosity which is applicable to a randomly branched polymer. Such a study is reported in this paper.

We synthesized a randomly branched polystyrene with a known degree of branching by copolymerization of styrene with 1,4-divinyl2,3,5,6-tetrachlorobenzene and prepared a series of narrow fractions by column fractionation. Then, using these fractionated samples as model polymers, we studied the effect of chain branching on the intrinsic viscosity and sedimentation coefficient. A study along the same line was performed by Thurmond and Zimm about twenty years ago. ${ }^{2}$ However, their result was less conclusive than was the present, mainly because of the lack of powerful fractionation techniques at that time.

\section{EXPERIMENTAL}

\section{Monomers}

A commercial styrene was washed three times with $5-\%$ aqueous $\mathrm{NaOH}$ to remove the inhibitor, dried over $\mathrm{CaCl}_{2}$, and kept in refrigerator. Before the monomer was used for polymerization, it was purified by distillation under reduced pressure.

1,4-Divinyl-2, 3, 5, 6-tetrachlorobenzene (hereafter abbreviated as DVTCB) as comonomer was synthesized in this laboratory from 1,4diethylbenzene through the following three processes: i.e., 1,4-diethylbenzene $\rightarrow$ 1, 4-diethyl2,3,5,6-tetrachlorobenzene $\rightarrow$ 1,4-bis(monobromoethyl)-2,3,5,6-tetrachlorobenzene $\rightarrow$ DVTCB. We followed the procedure by Harvey, et al., ${ }^{6}$ in the first process for chlorination, and the procedures by Ross, et al., ${ }^{7}$ in the remaining two processes. The details of these procedures are not described here. The final product, DVTCB, was yellowish white needles whose melting point was $86-88^{\circ} \mathrm{C}$. The overall yield was about $10 \%$, but no special effort was made to improve it.

\section{Polymerization}

The combination of styrene and DVTCB as comonomers was selected for three reasons. First, the physical properties of linear polystyrene have been most extensively studied in comparison with those of other polymers. Therefore, a good standard from which the branching effect is to be measured is available in this case. Secondly, the chemical analysis of chlorine content gives a direct information on the amount of DVTCB involved in a given polymer sample. This simplifies the kinetic estimation of the number of branch points in a molecule. Finally, the monomer reactivity ratio of styrene $r_{1}\left(=k_{11} / k_{12}\right)$ has been reported as 2.62 which is not much different from two. ${ }^{7}$ Thus, we can assume that styrene and DVTCB are built in the copolymer with an approximately equal probability. This is essential to obtain a polymer with uniform density of chain branching. In addition, the monomer reactivity ratio $r_{2}$ for DVTCB was found to be practically zero. $^{7}$ The occurrence of divinyl-divinyl sequence is thus avoided in this copolymer, and the probability of ring formation is largely diminished. All these facts indicate that the selected pair of comonomers is one of the best to obtain a model sample of randomly branched polymers.

Under the circumstances as stated above, the conversion of monomers $\theta$ may be written as

$$
\theta=\left(n_{1}+n_{2}\right) /\left(N_{1}+N_{2}\right) \simeq n_{1} / N_{1} \simeq n_{2} / N_{2}
$$

where $n_{1}$ represents the number of styrene molecules which have polymerized out of a total of $N_{1}$ in the system, and $n_{2}$ represents that of DVTCB molecules which have polymerized out of a total of $N_{2}$. The density of branch points $\rho$ is then given as

$$
\rho=\nu / n_{1}=-C[1+(1 / \theta) \ln (1-\theta)]
$$


Hydrodynamic Properties of Branched Polymers

Table I. Hydrodynamic properties of branched polystyrenes, A-series, in theta solvent, cyclohexane at $35^{\circ} \mathrm{C}$

\begin{tabular}{|c|c|c|c|c|c|c|c|c|c|c|}
\hline \multicolumn{2}{|c|}{ Fractionation } & \multirow{2}{*}{$\underset{\%}{W_{\mathrm{Cl}},}$} & \multirow{2}{*}{$\begin{array}{c}M_{n} \\
\times 10^{-4}\end{array}$} & \multirow{2}{*}{$\begin{array}{c}M_{v^{\mathrm{a}}} \\
\times 10^{-4}\end{array}$} & \multirow{2}{*}{$\frac{M_{w}}{M_{n}}$} & \multirow{2}{*}{$\begin{array}{l}{[\eta]_{\theta},} \\
\mathrm{d} l / \mathrm{g}\end{array}$} & \multirow{2}{*}{$k^{\prime} \theta$} & \multirow{2}{*}{$\begin{array}{c}s^{\circ} \theta \times 10^{13} \\
\sec \end{array}$} & \multirow{2}{*}{$\begin{array}{l}k_{s \theta} \\
\mathrm{d} l / \mathrm{g}\end{array}$} & \multirow{2}{*}{$\begin{array}{r}\beta_{\theta} \\
\times 10^{-6}\end{array}$} \\
\hline Code & Weight, $\mathrm{g}$ & & & & & & & & & \\
\hline A & 10.30 & 1.21 & 17.1 & - & - & 0.535 & 0.51 & - & - & - \\
\hline A- 1 & 0.520 & 1.16 & 4.36 & 4.70 & 1.08 & 0.164 & 0.52 & 3.54 & 0.18 & 2.32 \\
\hline A- 2 & 0.832 & 1.15 & 7.11 & 7.97 & 1.12 & 0.229 & 0.68 & 5.10 & 0.32 & 2.62 \\
\hline A- 3 & 1.070 & 1.07 & 12.4 & 13.9 & 1.12 & 0.300 & 0.52 & 6.50 & 0.43 & 2.52 \\
\hline A- 4 & 1.078 & 1.12 & 18.9 & 21.0 & 1.11 & 0.335 & 0.70 & 8.15 & 0.41 & 2.48 \\
\hline A- 5 & 0.989 & 1.14 & 30.0 & 38.7 & 1.29 & 0.386 & 0.57 & 12.4 & 0.49 & 2.62 \\
\hline A- 6 & 1.104 & 1.15 & 56.7 & 60.8 & 1.07 & 0.466 & 0.67 & 15.6 & 0.45 & 2.62 \\
\hline A- 7 & 0.963 & 1.21 & 114 & 155 & 1.36 & 0.571 & 0.54 & 27.5 & 0.58 & 2.64 \\
\hline A- 8 & 0.636 & 1.16 & 175 & 350 & 2.00 & 0.672 & 0.69 & 43.6 & 0.69 & 2.57 \\
\hline A- 9 & 1.308 & 1.16 & - & - & - & 0.927 & 0.51 & - & - & - \\
\hline A-10 & 0.613 & 1.16 & - & - & - & - & - & - & - & - \\
\hline
\end{tabular}

a Determined in methyl ethyl ketone.

$$
C=\left(k_{12}{ }^{\prime} / 2 k_{12}\right)\left(N_{2} / N_{1}\right)
$$

where $\nu$ is the total number of branch points in the system, $k_{12}$ is the reactivity of the first DVTCB bond, and $k_{12}{ }^{\prime}$ is that of the second.

We prepared two samples of copolymer, A and $\mathrm{B}$. In the preparation of the first sample designated as $\mathbf{A}$, polymerization mixture contained $147 \mathrm{~g}$ of styrene, $3.0 \mathrm{~g}$ of DVTCB, $0.144 \mathrm{~g}$ of $\alpha, \alpha^{\prime}$-azobisisobutyronitrile (hereafter abbreviated as AIBN), and $147 \mathrm{ml}$ of benzene as diluent. Accordingly, the molar ratio of styrene to DVTCB in the mixture, $N_{1} / N_{2}$, was 126. Polymerization was made at $60^{\circ} \mathrm{C}$ in a rotary thermostated bath for $46 \mathrm{hr}$. The conversion was 0.40 . When the polymerization time was prolonged under the same condition as above, gelation occurred at about $116 \mathrm{hr}$.

The preparation of the second sample $B$ was made in a larger scale. The polymerization mixture consisted of $685 \mathrm{~g}$ of styrene, $14.0 \mathrm{~g}$ of DVTCB, $0.670 \mathrm{~g}$ of AIBN and $685 \mathrm{ml}$ of benzene, hence the ratio of two comonomers $N_{1} / N_{2}$ was again 126 . Polymerization was made at $60^{\circ} \mathrm{C}$ for $46 \mathrm{hr}$, but the conversion was 0.384 , instead of 0.40 for the sample A.

Now, assuming that $k_{12}{ }^{\prime}=k_{12}$, and substituting the above values of $N_{1} / N_{2}$ and $\theta$ into eq 4 and 5 , we find

$\rho=0.00110$ (for A) and 0.00104 (for B)

The chlorine content $W_{\mathrm{Cl}}$ (in wt $\%$ ) and the degree of branching $\lambda$ may be defined as

$$
\begin{gathered}
W_{\mathrm{Cl}}=\left(n_{2} / n_{1}\right)\left(400 M_{\mathrm{C} 1} / M_{0}\right)=\left(N_{2} / N_{1}\right)\left(400 M_{\mathrm{Cl} 1} / M_{0}\right) \\
\lambda=1 / M_{\mathrm{b}}=\rho / M_{0}
\end{gathered}
$$

where $M_{\mathrm{b}}$ is the molecular weight per a branch point, $M_{0}$ is the monomer weight and $M_{\mathrm{Cl}}$ is the atomic weight of chlorine. In the present case, $M_{0}$ is approximately 104 , hence we obtain

$$
\begin{gathered}
W_{\mathrm{C} 1}=1.1 \%, \lambda=1.06 \times 10^{-5} \text { and } \\
M_{\mathrm{b}}=0.95 \times 10^{5} \quad(\text { for } \mathrm{A}) \\
W_{\mathrm{C} 1}=1.1 \%, \lambda=1.00 \times 10^{-5} \text { and } \\
M_{\mathrm{b}}=1.00 \times 10^{5} \quad(\text { for } \mathrm{B})
\end{gathered}
$$

This value of $W_{\mathrm{C} 1}$ is in close agreement with the analytical values shown in Table I.*

\section{Fractionation}

The branched polystyrene sample A was fractionated into ten fractions, A-1 to A-10, in a large-scale Desreux column at $30^{\circ} \mathrm{C}$ by using benzene-ethanol mixture as the eluting solvent. The flow rate was maintained at about $2 \mathrm{ml} / \mathrm{min}$, and the polymer concentration in the eluted solution was kept lower than 0.1 wt $\%$ during the experiment. After the tenth fraction was

* The use of the literature value ${ }^{5} r_{1}=2.6$, instead of 2.0, leads to

$$
W_{\mathrm{Ci}}=0.9 \%, \lambda=0.64 \times 10^{-5} \text { and } M_{\mathrm{b}}=1.54 \times 10^{5} \quad\left(9^{\prime}\right)
$$

This modification is rather in the wrong direction. 
M. Kurata, M. Abe, M. Ifama, and M. Matsushima

Table II, Hydrodynamic properties of branched polystyrenes, B-series

\begin{tabular}{|c|c|c|c|c|c|c|c|c|}
\hline \multicolumn{2}{|c|}{ Fractionation } & \multirow{2}{*}{$\begin{array}{l}M_{n} \\
\times 10^{-4}\end{array}$} & \multirow{2}{*}{$\begin{array}{c}M_{w} \\
\times 10^{-4}\end{array}$} & \multirow{2}{*}{$\frac{M_{w}}{M_{n}}$} & \multirow{2}{*}{$\begin{array}{c}[\eta]]_{\theta} \\
\mathrm{d} l / \mathrm{g}\end{array}$} & \multirow{2}{*}{$k^{\prime} \theta$} & \multirow{2}{*}{$\begin{array}{c}{[\eta], \mathrm{a}} \\
\mathrm{d} l / \mathrm{g}\end{array}$} & \multirow{2}{*}{$k^{\prime a}$} \\
\hline Code & Weight, $g$ & & & & & & & \\
\hline B & 210.55 & - & - & - & - & - & - & - \\
\hline B- 1 & 8.18 & 4.19 & - & - & 0.147 & 0.62 & 0.207 & 0.42 \\
\hline B- 2 & 14.60 & 7.42 & $8.96^{\mathrm{a}}$ & 1.21 & 0.219 & 0.52 & 0.360 & 0.36 \\
\hline B- 3 & 12.92 & 11.6 & $11.8^{\mathrm{b}}$ & 1.02 & 0.269 & 0.54 & 0.461 & 0.43 \\
\hline B- 4 & 15.75 & 18.8 & $18.9^{\mathrm{b}}$ & 1.01 & 0.319 & 0.53 & 0.627 & 0.31 \\
\hline B- 5 & 13.24 & 23.8 & $27.2^{\mathrm{c}}$ & 1.14 & 0.389 & 0.62 & 0.776 & 0.37 \\
\hline B- 6 & 11.85 & 35.4 & $39.9^{\mathrm{c}}$ & 1.13 & 0.417 & 0.55 & 0.948 & 0.37 \\
\hline B- 7 & 16.00 & 53.5 & $64.2^{\mathrm{c}}$ & 1.20 & 0.462 & 0.52 & 1.15 & 0.37 \\
\hline B- 8 & 15.26 & 78.0 & $125^{\mathrm{c}}$ & 1.60 & 0.518 & 0.57 & 1.50 & 0.40 \\
\hline B- 9 & 16.36 & 156 & $307^{c}$ & 1.97 & 0.606 & 0.58 & 2.16 & 0.45 \\
\hline B-10 & 14.75 & - & - & - & - & - & - & - \\
\hline B-11 & 14.24 & - & - & - & - & - & - & - \\
\hline B-12 & 12.58 & - & - & - & - & - & - & - \\
\hline B-13 & 24.68 & - & - & - & - & - & - & - \\
\hline
\end{tabular}

a Determined in toluene at $30^{\circ} \mathrm{C}$. ${ }^{\mathrm{b}}$ Determined in methyl ethyl ketone.

c Determined in three solvents, cyclohexane, methyl ethyl ketone, and toluene.

eluted, the column was clogged with gel component of the polymer. Thus, the recovery of polymers was stopped at $88.5 \mathrm{wt} \%$ of a total of $10.3 \mathrm{~g}$. These polymers were freeze-dried from benzene solutions. The results of the fractionation are summarized in Table I. The chlorine contents of all fractions were determined by the chemical analysis. As shown in the third column of Table I, they were constant within experimental error and in close agreement with the calculated value given in eq 9. The uniform distribution of branch points was thus assured experimentally for these samples.

Fractionation of the sample $B$ was made in a Baker-Williams column with giving a temperature gradient from 10 to $30^{\circ} \mathrm{C}$. Twelve fractions, B-1 to B-12, were obtained as shown in Table II. The chlorine content of the unfractionated sample was $1.1 \mathrm{wt} \%$ in agreement with eq 9.

\section{Linear Polystyrene}

The linear polystyrenes designated as L-series were purchased from the Pressure Chemical Company (Ohio, U.S.A.). These were anionically polymerized samples with narrow distribution of molecular weights.

\section{Theta Solvent}

Reagent grade cyclohexane was dried over a molecular sieve of $4 \mathrm{~A}$ after the removal of a trace of benzene by a silica gel column, and was fractionally distilled with metallic sodium. The theta temperature was found to be $34.7^{\circ} \mathrm{C}$ in cyclohexane by light-scattering measurements on the unfractionated sample A. This condition of the theta state is practically the same as that for linear polystyrene. This is quite natural, since the content of DVTCB is low in our sample.

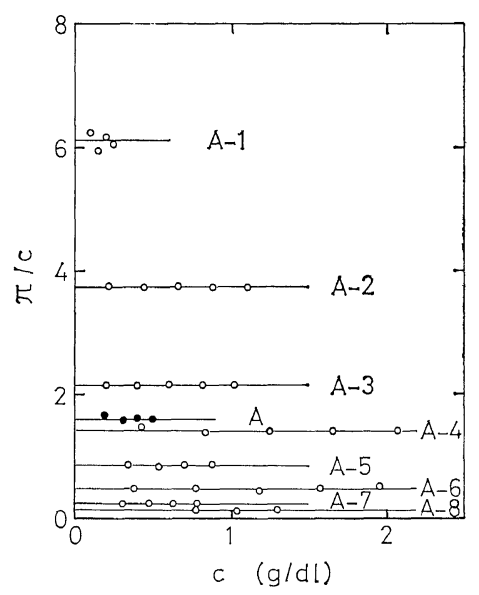

Figure 1. Reduced osmotic pressure $\pi / c$ plotted against polymer concentration $c$ in cyclohexane at $35^{\circ} \mathrm{C}$ : closed circles, unfractionated sample $\mathrm{A}$; open circles, fractionated samples A-1 to A-8. 
Table III. Hydrodynamic properties of linear polystyrenes, L-series.

\begin{tabular}{|c|c|c|c|c|c|c|c|c|c|}
\hline \multirow{2}{*}{ Code $^{a}$} & \multirow{2}{*}{$\begin{array}{c}M_{n} \times 10^{-4} \\
\text { Obsd }^{\mathrm{b}}\end{array}$} & \multicolumn{2}{|c|}{$M_{w} \times 10^{-4}$} & \multicolumn{2}{|c|}{$M_{w} / M_{n}$} & \multirow{2}{*}{$\begin{array}{l}{[\eta]_{\theta},} \\
\mathrm{d} l / \mathrm{g}\end{array}$} & \multirow{2}{*}{$k_{\theta}^{\prime}$} & \multirow{2}{*}{$\begin{array}{l}{[\eta]^{\mathrm{e}}} \\
\mathrm{d} l / g\end{array}$} & \multirow{2}{*}{$k^{\prime c}$} \\
\hline & & Obsd $^{b}$ & Nominal & Obsd & Nominal & & & & \\
\hline L- 7 & 4.9 & - & 5.1 & - & $\leq 1.06$ & 0.192 & 0.51 & 0.295 & 0.32 \\
\hline L- 1 & 15.7 & 16.5 & 16.0 & 1.05 & $\leq 1.06$ & 0.344 & 0.51 & 0.668 & 0.34 \\
\hline L- 3 & 40.1 & 44.4 & 41.1 & 1.11 & $\leq 1.06$ & 0.547 & 0.51 & 1.32 & 0.34 \\
\hline L- 6 & 72.5 & 87.6 & 86.0 & 1.21 & $\leq 1.15$ & 0.776 & 0.59 & 2.13 & 0.35 \\
\hline L-14 & - & 189 & 180 & - & $\leq 1.20$ & 1.14 & 0.58 & 3.62 & 0.34 \\
\hline
\end{tabular}

a These codes correspond to the producer's sample code, 7a, 1a, 3a, 6a, and 14a, respectively.

b Determined in three solvents, cyclohexane, methyl ethyl ketone, and toluene.

c Determined in toluene at $30^{\circ} \mathrm{C}$.

\section{Osmotic Pressure}

Osmotic-pressure measurements were performed for the unfractionated sample $A$ and eight fractionated samples A-1 to A-8 in cyclohexane at $35^{\circ} \mathrm{C}$ by using a Mechrolab high speed membrane osmometer fitted with a gel cellophane as semipermeable membrane. Figure 1 illustrates the concentration dependence of the osmotic pressure $\pi$, where the open circles represent the experimental data. These data indicate that the theta temperature is common to all the samples studied. This may be counted as an additional evidence for uniform composition of comonomers in the samples, hence for uniform distribution of branch units. The number-average molecular weights $M_{n}$ obtained are shown in Table I.

The osmotic-pressure measurements for the samples of B- and L-series were made in toluene at $37^{\circ} \mathrm{C}$. The values of $M_{n}$ obtained are given in Tables II and III.

\section{Light Scattering}

Light-scattering measurements were performed for the samples, A-1 to $8, \mathrm{~B}-2$ to 9 , and L-1, 3,6 , and 14 , in methyl ethyl ketone at $30^{\circ} \mathrm{C}$ by using a Shimadzu photoelectric photometer equipped with a cylindrical cell. For the samples from B-5 to B-9 and of L-series, the measurements were also performed in two other solvents, cyclohexane at $35^{\circ} \mathrm{C}$, and toluene at $30^{\circ} \mathrm{C}$. The refractive-index increments in the above three solvents are $0.235,0.181$ and 0.120 $\mathrm{ml} / \mathrm{g}$, respectively, for the light of $436-\mathrm{m} \mu$ wavelength. Measurements of the scattered-light intensity were carried out on at least four concentrations in the range of $0.003-0.002 \mathrm{~g} / \mathrm{ml}$ and at nine scattering angles between $35^{\circ}$ and $145^{\circ}$. The details of the measurements have been described in an earlier paper. ${ }^{8}$

The weight-average molecular weight $M_{w}$, second virial coefficient $A_{2}$, and $z$-average meansquare radius of gyration $\left\langle S^{2}\right\rangle_{z}$ of each sample were determined by the ordinary procedure due to Zimm. The values of $M_{w}$ are listed in Tables I, II, and III, while those of $A_{2}$ and $\left\langle S^{2}\right\rangle_{z}$ in Table IV.* The nominal values of $M_{w}$ for the samples of L-series given by the producer are also listed in Table III, which are in agreement with our observed values. The observed ratio $M_{w} / M_{n}$ was about 1.1 for the samples A-1 to 4 and B-2 to 6 , indicating that the fractionation was efficiently achieved in this range of molecular weight. In the range of higher molecular weight, the efficiency of fractionation was somewhat lowered, but still satisfactory. Using these figures of $M_{w} / M_{n}$ and assuming the Schulz-type or the log-normal distribution of $M$, we estimated roughly the $z$ average molecular weight $M_{z}$ of each sample. The results are shown in parentheses in Table IV.

\section{Intrinsic Viscosity}

Dilution viscometers of the Ubbelohde type were used for measurements of intrinsic viscosity. Corrections for the kinetic energy and

* The data on the samples of B- and L-series have been partly published in an earlier paper. $^{8}$ However, the values of $M_{w}$ and $A_{2}$ reported there should be modified by a factor of $0.845,0.760$, and 0.866 in methyl ethyl ketone $\left(30^{\circ} \mathrm{C}\right)$, cyclohexane $\left(35^{\circ} \mathrm{C}\right)$, and toluene $\left(30^{\circ} \mathrm{C}\right)$, respectively, because of the use of erroneous calibration constants. 
Table IV. Light scattering data of linear and branched polystyrenes, A-, B-, and L-series.

\begin{tabular}{cccccc}
\hline Sample code & $M_{w} \times 10^{-4}$ & $M_{z} \times 10^{-4}$ & $\begin{array}{c}\left\langle S^{2}\right\rangle \theta^{1 / 2} \\
\times 10^{8}, \mathrm{~cm}\end{array}$ & $\begin{array}{c}A_{2} \times 10^{4 \mathrm{a}}, \\
\mathrm{mol} \mathrm{g}^{-2} \mathrm{~cm}^{3}\end{array}$ & $\begin{array}{c}\left\langle S^{2}\right\rangle z^{1 / 2 \mathrm{a}} \\
\times 10^{8}, \mathrm{~cm}\end{array}$ \\
\hline A- 4 & 21.0 & $(23.0)$ & - & 1.28 & 128 \\
A- 5 & 38.7 & $(43.0)$ & - & 2.08 & 191 \\
A- 6 & 60.8 & $(68.0)$ & - & 0.89 & 223 \\
A- 7 & 155 & $(195)$ & - & 0.81 & 352 \\
A- 8 & 350 & $(590)$ & - & 0.56 & 455 \\
B- 4 & 18.9 & $(21.0)$ & - & 1.83 & 101 \\
B- 5 & 27.2 & $(30.0)$ & 149 & 1.94 & 142 \\
B- 6 & 39.9 & $(44.0)$ & 170 & 1.37 & 179 \\
B- 7 & 64.2 & $(75.0)$ & 205 & 1.04 & 268 \\
B- 8 & 125 & $(190)$ & 257 & 0.92 & 323 \\
B- 9 & 307 & $(520)$ & 395 & 0.58 & 512 \\
L- 1 & 16.5 & $(17.0)$ & 110 & 1.85 & 114 \\
L- 3 & 44.4 & $(45.0)$ & 177 & 1.56 & 219 \\
L- 6 & 87.6 & $(96.0)$ & 277 & 1.38 & 334 \\
L-14 & 189 & $(220)$ & 391 & 0.96 & 524 \\
\hline
\end{tabular}

a Determined in methyl ethyl ketone at $30^{\circ} \mathrm{C}$.

shear rate effect were both negligible. Extrapolation of the viscosity data to infinite dilution was made by using two plots, $\eta_{\mathrm{sp}} / \boldsymbol{c} v s . \eta_{\mathrm{sp}}$ and $\left(\ln \eta_{\mathrm{r}}\right) / c v s . c$, where $\eta_{\mathrm{sp}}$ and $\eta_{\mathrm{r}}$ denote the specific viscosity and relative viscosity of a solution of concentration $c$, respectively. The Huggins constant $k^{\prime}$ was estimated from the slope of the $\eta_{\mathrm{sp}} / c \boldsymbol{c} s . \eta_{\mathrm{sp}}$ plot.

The measurements were made both in cyclohexane at $35^{\circ} \mathrm{C}$ and in toluene at $30^{\circ} \mathrm{C}$. The results are shown in Tables I, II, and III.

\section{Sedimentation Coefficient}

Sedimentation-velocity measurements were carried out on the samples of A-series in a Spinco model E ultracentrifuge equipped with an RTIC temperature control unit. The solvent used was cyclohexane. In all the measurements, temperature was controlled at $35.0 \pm 0.1^{\circ} \mathrm{C}$. The rotor speed employed was $59780 \mathrm{rpm}$ for fractions A-1 to 4, $42040 \mathrm{rpm}$ for fractions A-5 to 7 and $29500 \mathrm{rpm}$ for fraction A-8. Since the Schlieren diagrams were more or less distorted from the symmetric gaussian form, the boundary positions were determined by the second moment method. ${ }^{9}$ The sedimentation coefficient at zero concentration and one atmosphere $s^{0}$ was determined by the procedures established by our group. ${ }^{10}$ The results are shown in
Table I where the values of the concentration dependence coefficient $k_{\mathrm{s}}$ are also tabulated.

\section{RESULTS AND DISCUSSIONS}

\section{Intrinsic Viscosity in Theta Solvent}

The effect of long-chain branching on the intrinsic viscosity has been theoretically investigated for two types of branching, the star type ${ }^{4}$ and comb type. ${ }^{5}$ These theories give

$$
\begin{aligned}
& {[\eta]_{\theta \mathrm{b}}=G[\eta]_{\theta l} } \\
G=g^{1 / 2} & \text { (star type }) \\
= & g^{3 / 2} \quad(\text { comb type })
\end{aligned}
$$

where $[\eta]_{\theta b}$ is the intrinsic viscosity of a branched polymer of molecular weight $M$ in a theta solvent and $[\eta]_{\theta l}$ is that of a linear polymer of the same molecular weight. $g$ is the contraction factor of the mean-square radius of gyration $\left\langle S^{2}\right\rangle$ as defined by eq 1 .

In the case of randomly branched polymers with tri- or tetra-functional branching units, the theory of Zimm and Stockmayer gives ${ }^{1}$

$$
\begin{aligned}
& g_{3}=\left[(1+\lambda M / 7)^{1 / 2}+(4 / 9 \pi) \lambda M\right]^{-1 / 2} \\
& g_{4}=\left[(1+\lambda M / 6)^{1 / 2}+(4 / 3 \pi) \lambda M\right]^{-1 / 2}
\end{aligned}
$$

which are applicable to fractionated samples with narrow distribution of molecular weight. 


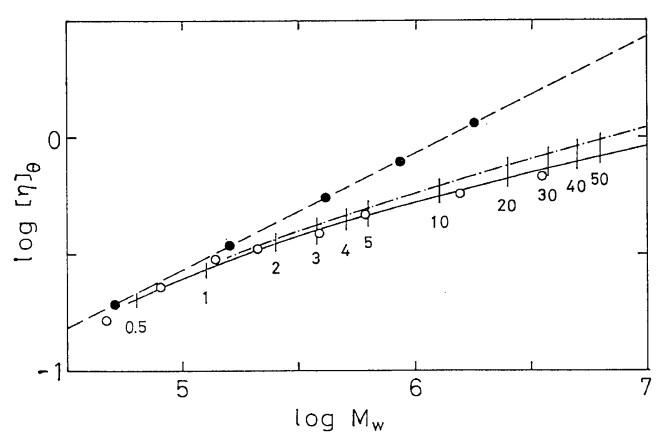

Figure 2. Intrinsic viscosities in theta solvent, cyclohexane at $35^{\circ} \mathrm{C}$ : closed circles, observed values for linear samples, L-series; open circles, observed values for branched samples, A-series; broken line, eq 15 ; solid curve, calculated values of $[\eta] \theta \mathrm{b}$ based on eq 10,15 , and 17 with $\lambda=0.80 \times 10^{-5}$; chain curve, calculated values of $[\eta]_{\theta \mathrm{b}}$ based on eq 10 , 15 and $G_{4}=g_{4}{ }^{1 / 2}$ with $\lambda=0.80 \times 10^{-5}$. Numbers attached to vertical bars represent the values of $m=\lambda M$.

The subscripts attached to $g$ denote the functionality of branch units. Theoretical expression for $G$ is not available as yet. However, it has been suggested that the hydrodynamic behavior of the randomly branched molecules has a close resemblance to that of the star molecules. ${ }^{5}$ Accordingly, we may assume

$$
G=g^{\nu}
$$

where $\nu$ is a constant roughly equal to 0.5 .

Figure 2 shows the double logarithmic plots of $[\eta]_{\theta}$ against $M_{w}$, where the data for the branched samples of A-serries are shown by open circles and those for the linear samples of L-series by closed circles. The broken line represents the relationship,

$$
[\eta]_{\theta l}=8.50 \times 10^{-4} M_{w}^{0.5 *}
$$

which were presented for linear narrow-distribution polystyrenes by Berry. ${ }^{11}$ This equation is practically identical to the equation of Cowie and Bywater, ${ }^{12}$ i.e., $[\eta]_{\theta l}=8.60 \times 10^{-4} M_{w}{ }^{0.50}$.

The calculation of $[\eta]_{\theta \mathrm{b}}$ was attempted with two values of $\nu$, i.e., 0.6 and 0.5 . With the former value of $\nu$, we can put

$$
\begin{aligned}
& G_{3}=g_{3}{ }^{0.6}=\left[(1+\lambda M / 7)^{1 / 2}+(4 / 9 \pi) \lambda M\right]^{-0.3} \\
& G_{4}=g_{4}{ }^{0.6}=\left[(1+\lambda M / 6)^{1 / 2}+(4 / 3 \pi) \lambda M\right]^{-0.3}
\end{aligned}
$$

depending on the functionality of the branch units. Then, using eq 10, 15, and 17, and putting $\lambda=0.80 \times 10^{-5}$, we obtain the calculated values of $[\eta]_{\theta b}$ which are shown in Figure 2 by the solid curve. The vertical bar represents the molecular weight of the polymer which involves on the average the indicated number of branch units, $m=\lambda M$, in a molecule. On the other hand, with the second choice that $\nu=0.5$, we obtain the values of $[\eta]_{\theta \mathrm{b}}$ indicated by the chain curve in the figure. Clearly, better agreement with the observed values is obtained with the first choice of $\nu$.

With the choice of $\nu=0.6$, the $G$-factor becomes asymptotically proportional to $M^{-0.30}$ as $M$ increases, and hence $[\eta]_{\theta \mathrm{b}}$ to $M^{0.20}$. This prediction can be favorably compared with the experimental facts that the intrinsic viscosities of highly branched dextran ${ }^{13}$ and polymethylsiloxane $^{14}$ are proportional to $M_{w}^{0.20}$, rather than $M_{w}{ }^{0.25}$, in theta solvents, water at $25^{\circ} \mathrm{C}$ and methyl ethyl ketone at $20^{\circ} \mathrm{C}$, respectively.

Figure 3 represents a test of sensitivity of the calculated value of $[\eta]_{\theta \mathrm{b}}$ to the assumed value of $\lambda$. The solid curve is the reproduction of the curve given in Figure 2 and it corresponds to the choice that $\lambda=0.80 \times 10^{-5}$. On the other hand, two dotted curves correspond to $\lambda=0.60$ $\times 10^{-5}$ and $1.00 \times 10^{-5}$, respectively. It may be concluded from this analysis that the estimation of $\lambda$ is possibly achieved with the accuracy of $15-20 \%$ and hence $\lambda=\left(0.80_{ \pm} 0.15\right) \times 10^{-5}$. This result is to be compared with the kinetic esti-

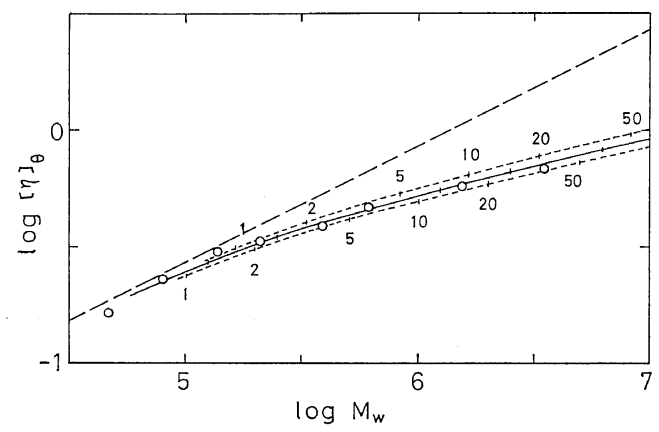

Figure 3. Calculated values of $[\eta]_{\theta \mathrm{b}}$ corresponding to three different choices of $\lambda$-value: solid curve, $\lambda=0.80 \times 10^{-5}$; upper dotted curve, $\lambda=0.60$ $\times 10^{-5}$; lower dotted curve, $\lambda=1.00 \times 10^{-5}$. Broken line and open circles are the same as in Figure 2. 


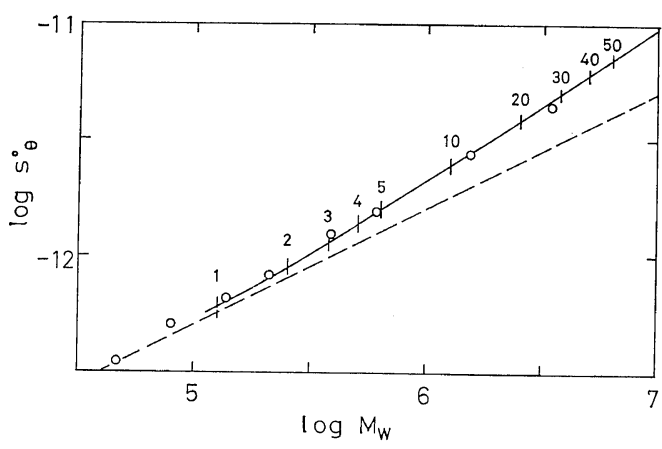

Figure 4. Sedimentation coefficients in theta solvent, cyclohexane at $35^{\circ} \mathrm{C}$ : open circles, observed values for branched samples, A-series; broken line, eq 18; solid curve, calculated values based on eq 18,20 , and 21 with $\lambda=0.80 \times 10^{-5}$. Numbers attached to vertical bars represent the values of $m=\lambda M$.

mate given in eq 9. A small difference existing between these two estimates of $\lambda$ may be attributable to the possible inequality between $k_{12}^{\prime}$ and $k_{12}$.

The experimental data of $[\eta]_{\theta \mathrm{b}}$ for the samples of B-series were also analysed by the same method as above, though not reproduced here. The same estimate of $\lambda$ was obtained as expected.

\section{Sedimentation Coefficient in Theta Solvent}

The double logarithmic plot of $s_{\theta}{ }^{0}$ against $M_{w}$ is shown in Figure 4, where the open circles represent the experimental data. The broken line represents the relationship,

$$
s_{\theta l}^{0}=1.60 \times 10^{-15} M_{w}^{0.50}
$$

which is only slightly different from the equation of Cowie and Bywater, ${ }^{12}$ i.e., $s_{\theta l}^{0}=1.50$ $\times 10^{-15} M_{w}{ }^{0.50}$. The Mandelkern and Flory constant $\beta$ is defined by

$$
\beta=\frac{s^{0}[\eta]^{1 / 3}}{M^{2 / 3}} \frac{N_{A} \eta_{0}}{\left(1-\bar{v} \rho_{0}\right)}
$$

where $N_{A}$ is the Avogadro number, $\eta_{0}$ is the solvent viscosity, $\rho_{0}$ is the solvent density and $\bar{v}$ is the specific volume of polymer. For the system of polystyrene in cyclohexane, $\eta_{0}=7.40$ $\times 10^{-3}$ poise, $\rho_{0}=0.7648 \mathrm{~g} / \mathrm{ml}$ and $\bar{v}=0.933 \mathrm{ml} / \mathrm{g}$ at $35^{\circ} \mathrm{C}$. Therefore, substituting eq 15 and 18 into eq 19 , we obtain $\beta=2.48 \times 10^{6}$.

A theory of the sedimentation coefficient of branched polymers has been developed by one of the present authors. ${ }^{5}$ In the case of a randomly branched polymer with tetra-functional branch units, this theory gives

$$
\begin{gathered}
s_{\theta \mathrm{b}}^{0}=h^{-1} s_{\theta l}^{0} \\
h^{-1}=0.605(\lambda M)^{1 / 4}+0.474(\lambda M)^{-1 / 4}
\end{gathered}
$$

Equation 21 is an approximate formula which is acurate to within $1 \%$ when $\lambda M \geq 2$. For smaller values of $\lambda M$, the numerical values of $h^{-1}$ have been given in the original paper. ${ }^{5}$ Now, putting again $\lambda=0.80 \times 10^{-5}$, we obtain the values of $s_{\theta \mathrm{b}}^{0}$ which are shown in Figure 4 by the solid curve. The number attached to each vertical bar represents the number of branch points per a molecule. Agreement between theory and experiment is again satisfactory.

The Flory-Mandelkern constants $\beta$ for the branched samples are also evaluated as shown in Table I. They are a little higher than the value for the linear sample.

\section{Intrinsic Viscosity in Good Solvent}

From a practical viewpoint, it is desirable that the estimation of $\lambda$ can be based on the intrinsic viscosities obtained in a good solvent rather than those obtained in the theta solvent. A test of this possibility is made by using the data obtained for the samples of B- and L-series in toluene at $30^{\circ} \mathrm{C}$. The result is illustrated in Figure 5, where the open circles represent the data for the branched samples and the closed

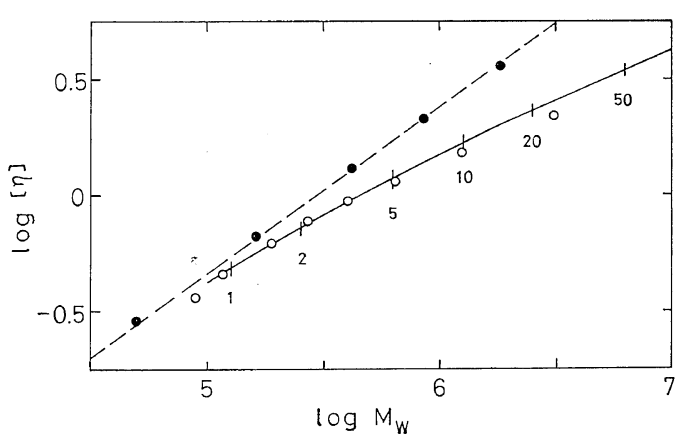

Figure 5: Intrinsic viscosities in toluene at $30^{\circ} \mathrm{C}$ : open circles, observed values for branched samples, B-series; closed circles, observed values for linear samples, L-series; broken line, eq 22; solid curve, calculated values of $[\eta]_{b}$ based on eq 17, 22, and 23 with $\lambda=0.80 \times 10^{-5}$. Numbers attached to vertical bars represent the values of $m=\lambda M$. 
circles those for the linear samples. The broken line represents the relationship,

$$
[\eta]_{l}=1.14 \times 10^{-4} M_{w}{ }^{0.72}
$$

while the solid curve represents the values of $[\eta]_{\mathrm{b}}$ calculated by

$$
[\eta]_{\mathrm{b}}=G_{4}[\eta]_{l}
$$

with eq 17, 22, and $\lambda=0.80 \times 10^{-5}$. Agreement between the observed and calculated values of $[\eta]_{\mathrm{b}}$ is satisfactory. Thus, we may conclude that the effect of excluded volume is of minor importance in the present problem and that a reasonable estimate of $\lambda$ can be based on the intrinsic viscosity in good solvent as well as on that in theta solvent.

\section{Functionality of Branch Points}

In the present case of branched polystyrene, we can readily identify the functionality of branch points to be four from a consideration of the polymerization mechanism. However, in general, this will not be the case, for the mechanism of chain branching often remains unclarified. Thus, it seems worthwhile to note that eq 16 and 17 are mutually superposable in the plot of $\log G$ against $\log \lambda$ to within a few percent error. Figure 6 illustrates this statement, where the solid line represents $\log G_{4}$ plotted against $\log (\lambda M)$, while the crosses represent $\log G_{3}$ plotted against $\log (\lambda M / 2.67)$. This implies that, in a wide range of $m=\lambda M$, one can utilize either of eq 16 and 17 to estimate the effect of chain branching on the

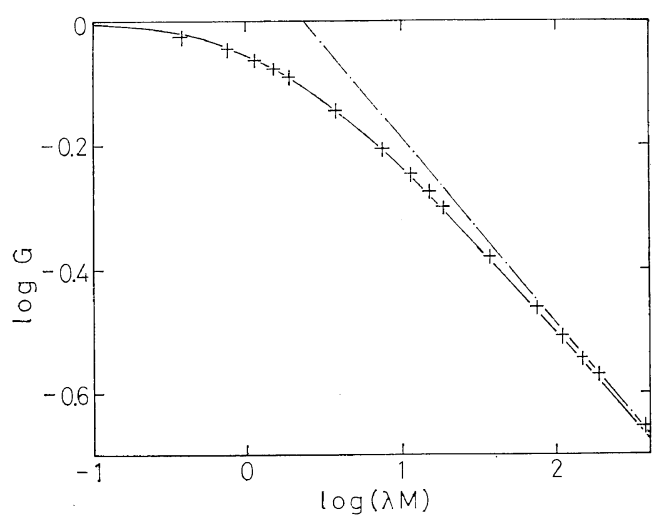

Figure 6. Viscosity branching factors $G$ plotted against $\lambda M$ : solid curve, $\log G_{4} v s . \log (\lambda M)$; chain line, eq 25 ; crosses, $\log G_{3} v s . \log (\lambda M / 2.67)$. intrinsic viscosity. The values of $\lambda$ obtained depend on the assumed value of functionality, but they are mutually convertible according to the relationship,

$$
\lambda_{3}=2.67 \lambda_{4}
$$

The chain line in Figure 6 represents the asymptotic equation of $G_{4}$ for large $\lambda M$,

$$
G_{4}=1.293(\lambda M)^{-0.3}
$$

\section{Mean-Square Radius of Gyration}

Finally, let us treat of the data on the meansquare radius of gyration $\left\langle S^{2}\right\rangle_{z}$ given in Table IV. The data for the linear polystyrenes, Lseries, are well represented by

$$
\begin{aligned}
& \left\langle S^{2}\right\rangle_{z \theta l}{ }^{1 / 2}=2.88 \times 10^{-9} M_{\cdot w}^{0.50} \quad[\mathrm{~cm}] \\
& \left\langle S^{2}\right\rangle_{z l}{ }^{1 / 2}=1.43 \times 10^{-9} M_{w}^{0.57} \quad[\mathrm{~cm}]
\end{aligned}
$$

The combination of eq 26 with eq 15 and of eq 27 with eq 22 gives $2.41 \times 10^{21}$ and $2.64 \times 10^{21}$, respectively, for the Flory universal constant $\Phi$.

Figures 7 and 8 shows the dobble logarithmic plots of $\left\langle S^{2}\right\rangle_{z \theta}{ }^{1 / 2}$ and $\left\langle S^{2}\right\rangle_{z}{ }^{1 / 2}$ against the molecular weight. In the figures, the broken lines represent the relationships eq 26 and eq 27, while the solid curves represent the values of $\left\langle S^{2}\right\rangle_{z \theta \mathrm{b}}{ }^{1 / 2}$ and $\left\langle S^{2}\right\rangle_{z \mathrm{~b}}{ }^{1 / 2}$ calculated by eq 1 and 2 with $\lambda=0.80 \times 10^{-5}$. If the data are plotted against $M_{w}$, the experimental points for the

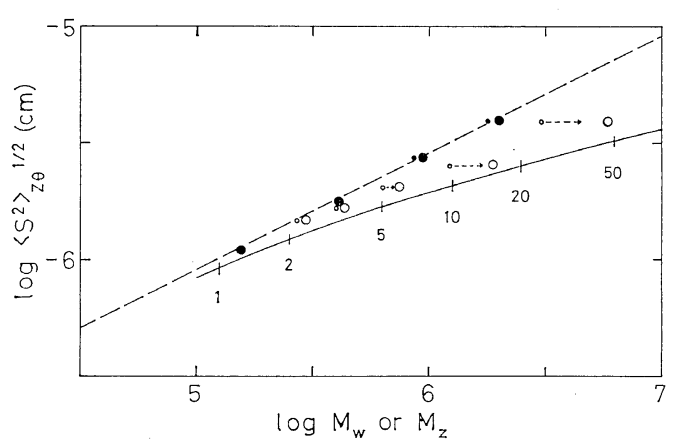

Figure 7. Root-mean-square radii of gyration in theta solvent, cyclohexane at $35^{\circ} \mathrm{C}$ : closed circles, observed values for linear samples, L-series; open circles, observed values for branched samples, B-series; broken line, eq 26; solid curve, calculated values for $\lambda=0.80 \times 10^{-5}$. The abscissa is $\log M_{w}$ for the circles of small size and $\log M_{z}$ for the circles of large size. Numbers attached to vertical bars represent the values of $\lambda M_{w}$ or $\lambda M_{z}$. 


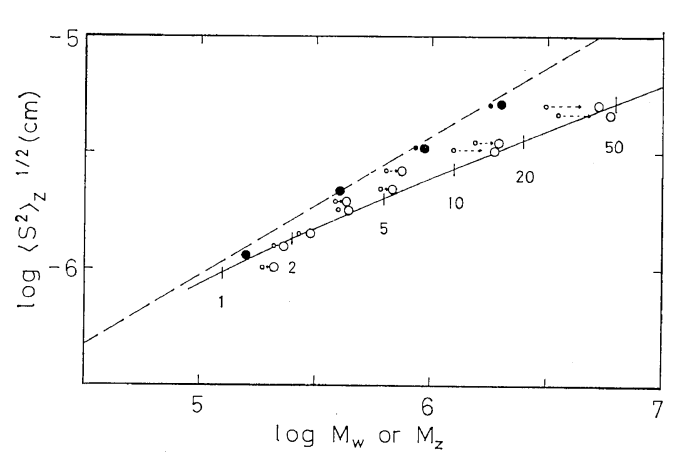

Figure 8. Root-mean-square radii of gyration in methyl ethyl ketone at $30^{\circ} \mathrm{C}$ : open circles, observed values for branched samples, A- and Bseries; broken line, eq 27 . Other symbols have the same meaning as those in Figure 7.

branched samples fall far above the theoretical curves as shown by the small open circles. The discrepancy is attributable to the effect of molecular weight distribution, and accordingly it is diminished by replotting the data against $M_{z}$ as illustrated by the large open circles. However, in view of rather large scattering of the data, it is unlikely that a reliable estimate of $\lambda$ can be obtained from $\left\langle S^{2}\right\rangle_{z}$, unless very accurate data for $M_{z}$ are available.

After this work was completed, a paper on the hydrodynamic properties of a branched polymer, poly(methyl methacrylate), was published by Kamada and Sato. ${ }^{15}$ Their results were generally in agreement with ours, though they recommended the $g^{1 / 2}$-rule, instead of the present $g^{0.6}$-rule, to represent the branching effect on $[\eta]$.

Acknowledgement. The main part of this study was worked out in the period 1966-1967 when one of the authors (M.A.) was a senior scientist at Kyoto University on leave of absence from the Japan Synthetic Rubber Company. We wish to thank Dr. Terutake Homma of JSR for making this arrangement and for many stimulating discussions. This study was supported in part by the Japan Synthetic Rubber Company, Ltd., and the International Institute of Synthetic Rubber Producers, Inc., under contracts with the Kyoto University.

\section{REFERENCES}

1. B. H. Zimm and W. H. Stockmayer, J. Chem. Phys., 17, 1301 (1949).

2. R. W. Kilb, J. Polym. Sci., 38, 403 (1959).

3. C. D. Thurmond and B. H. Zimm, ibid., 8, 477 (1952).

4. B. H. Zimm and R. W. Kilb, ibid., 37, 19 (1959).

5. M. Kurata and M. Fukatsu, J. Chem. Phys., 41, 2934 (1964).

6. P. G. Harvey, F. Smith, M. Stacey, and J. C. Tatlow, J. Appl. Chem., 1954, 325.

7. S. D. Ross, M. Markarian, H. H. Young, Jr., and M. Nazzewski, J. Amer. Chem. Soc., 72, 1133 (1950).

8. M. Abe, M. Iwama, and T. Homma, Kogyo Kagaku Zasshi, (J. Chem. Soc., Japan, Chem. Ind. Sect.), 72, 1572 (1969).

9. H. K. Schachman, "Ultracentrifugation in Biochemistry" Academic Press, New York, N. Y., 1964, p 65.

10. M. Abe, K. Sakato, T. Kageyama, M. Fukatsu, and M. Kurata, Bull. Chem. Soc. Japan, 41 2330 (1968).

11. G. C. Berry, J. Chem. Phys., 46, 1338 (1967).

12. J. M. Cowie and S. Bywater, Polymer, 6, 197 (1965).

13. G. Meyerhoff, Fortsch. Hochpolym. Forsch., 3, 59 (1961).

14. F. P. Price, S. G. Martin, and J. P. Bianchi, J. Polym. Sci., 22, 41 (1956).

15. K. Kamada and H. Sato, Polymer J., 2, 489, 593 (1971). 